\title{
Su un problema esterno per la equazione delle onde.
}

\author{
Memoria di Carlo Pucci (a Roma)
}

Sunto. - Si considera il problema di CACCHY per la equazione $u_{t t}=u_{x x}+u_{y y}$ con dati ini. ziali sui due piani caratteristici $t= \pm y$. Si dà un teorema di esistenza ed unicità della soluzione nella "regione esterna* $|t| \leq|y|$, e si prova che la solusione non dipende in modo continuo dai dati.

Snmmary. - We consider the CAUCHy problem for the wave equation $u_{t t}=u_{x_{x}}+u_{y y}$ with initial data on the characteristic planes $t= \pm y$. We discuss the problem related to the "exterior region" $|t| \leq|y|$; we prove uniqueness of the solution, its instability with respect to the data, and the existence of the solution if the data are analytic functions of $x$ (this hypothesis is essential, as we prove with an example).

\section{Introduzione.}

Consideriamo il problema consistente nella ricerca di una soluzione della equazione delle onde piane

$$
L u \equiv u_{t t}-u_{x x}-u_{y \nu}=0,
$$

soddisfacente a condizioni iniziali assegnate su due piani caratteristici

$$
u(x, y, y)=\varphi_{1}(x, y), \quad u(x, y,-y)=\varphi_{2}(x, y)
$$

con $\varphi_{1}(x, 0)=\varphi_{2}(x, 0)$. Si tratta dell'analogo nello spazio $(x, y, t)$ del problema di Goursat :

$$
u_{t t}-u_{y y}=0, \quad u(y, y)=\varphi_{1}(y), \quad u(y,-y)=\varphi_{2}(y)
$$

Se $\varphi_{1}$ e $\varphi_{2}$ sono di classe $C^{(2)}$ esiste una ed una sola soluzione del problema (1), (2) nella « regione interna» $|t| \geq|y|$ ed il valore della soluzione in un punto $P$ dipende soltanto dai valori di $\varphi_{1}$ e $\varphi_{2}$ nei punti appartenenti ai due semipiani $t=|y|$ ed interni al cono caratteristico di vertice $P\left({ }^{1}\right)$.

(1) Questo preblema ed il problema analogo con dati iniziali sul cono caratteristico $x^{2}+y^{2}=t^{2}$, anzichè su $y= \pm t$, sono stati trattati principalmente da V. VoLTERRA [8], R. D'adhimar [1], J. Hadamard [2], M. Riesz [3] e [4]. 
Per quanto riguarda la «regione esterna» $y \geq|t|$ è stato provato da A. A. Temixakov che esiste una ed una sola soluzione analitica nell' ipotesi che $\varphi_{1}$ e $\varphi_{2}$ siano analitiche in $x$ ed $y\left({ }^{2}\right)$.

In questa Memoria cerchiamo di svolgere una discussione esauriente del problema esterno ponendo in evidenza alcune notevoli diversità col corrispondente problema interno. Proviamo dapprima che esiste al più una soluzione di classe $C^{(2)}$ e che il valore della soluzione in un punto dipende dai valori assunti da $\varphi_{1}$ e $\varphi_{2}$ in un insieme di misura piana arbitrariamente piccola.

Proviamo poi l'instabilità della soluzione rispetto ai dati $\varphi_{1}$ e $\varphi_{2}$ e cioè che una variazione arbitrariamente piccola di $\varphi_{1}$ e $\varphi_{2}$ nella metrica naturale dello spazio $C^{(\prime)}(r$ numero naturale qualsiasi) può comportare una variazione arbitrariamente grande della soluzione. Questo è provato mediante la costruzione di una successione $\left\{u_{n} \mid\right.$ di soluzioni in tutto lo spazio della equazione delle onde piane tale che: $\left.1^{\circ}\right)\left\{u_{n}\right\}$ e le successioni delle derivate parziali fino ad un ordine $s$ fissato, comunque elevato, convergono uniformemente a zero nella "regione interna $>|y| \leq|t|, 2^{\circ}$ ) le funzioni $u_{n}$ non sono equilimitate in alcun sottoinsieme aperto della «regione esterna» $|y| \geq|t|$.

Proviamo nell' ultimo paragrafo un teorema di esistenza in ipotesi meno restrittive di quelle impiegate da TemLYakov supponendo l'analiticità di $\varphi_{1}$ e $\varphi_{2}$ solamente rispetto alla variabile $x$; stabiliamo anche una rappresentazione integrale della soluzione $e$ ne indichiamo una diversa rappresentazione in serie. Osserviamo infine che nel teorema di esistenza provato è essenziale l'ipotesi che $\varphi_{1}$ e $\varphi_{2}$ siano dotate di derivate di qualsiasi ordine rispetto ad $x$.

Ringrazio MAROEL RIESz per avermi suggerito lo studio di questo problema e JoAquim Diaz per alcune utili discussioni sull'argomento.

\section{Unicità della soluzione.}

Fissato un punto $P$ indichiamo con $P^{\prime}$ e $P^{\prime \prime}$ le proiezioni ortogonali di $P$ sui piani $y= \pm t$ e con $P^{\prime \prime}$ la proiezione di $P$ sull' asse $x$. Sia $A$ un insieme aperto convesso, tale che per ogni suo punto $P=(x, y, t)$ risulti $y>|t|$ e $P^{\prime}, P^{\prime \prime}, P^{\prime \prime} \in \bar{A}\left({ }^{3}\right)$.

Esiste al più una funzione $u$, di classe $O^{(2)}$ in $\bar{A}$, ed ivi soddisfacente alle (1), (2). Il valore di $u$ in $P$ è determinato univocamente dai valori assunti $d a \varphi_{1} e \varphi_{2}$ in un intorno comunque piccolo dei segmenti $P^{\prime} P^{\prime \prime \prime}$ e $P^{\prime \prime} L^{\prime \prime \prime}$ sui piani portanti $i$ dati.

La dimostrazione consiste in un riadattamento al caso considerato del

(2) Vedere [5] e, per una estensione, Tembyakox e Sohoar [7]. Altri problemi esterni per la equazione delle onde, di un tipo diverso da quello qui considerato, sono stati trattati da Volterra e Hadamard. Ofr. [2], pag. 220 e pagg. 270-272.

(3) Indichiamo con $\bar{A}$ la chiusura dell'insieme $A$. 
procedimento impiegato da HoLMGREN per provare l'unicità delle soluzioni di problemi di CaUCHY.

Fissato $P \equiv\left(x_{0}, y_{0}, t_{0}\right)$ in $A$ ed una costante positiva sufficientemente piccola, esiste un numero naturale $n$ tale che, indicato con $T$ l'insieme dei punti $(x, y, t)$ verificanti le limitazioni

$$
\left(\frac{x-x_{0}}{\varepsilon}\right)^{2 n}+\left(\frac{y+t+\varepsilon}{y_{0}+t_{0}+2 \varepsilon}\right)^{2 n}+\left(\frac{y-t+\varepsilon}{y_{0}-t_{0}+2 \varepsilon}\right)^{2 n}<1, \quad y>|t|,
$$

risulti $P \in T, T \subset A$. Ciò segue dalle ipotesi su $A$. Indichiamo con $B_{\varepsilon}$ l'intersezione di $\bar{T}$ con i piani $y= \pm t$; ogni punto di $B_{\varepsilon}$ dista da uno dei due segmenti $P^{\prime} P^{\prime \prime \prime}$ e $P^{\prime \prime} P^{\prime \prime \prime}$ per meno di $2 \varepsilon$.

Indichiamo con $u$ una funzione, di elasse $C^{(2)}$ in $\bar{A}$, soluzione dell' equazione delle onde, nulla su $B_{\varepsilon}$. Abbiamo dimostrato il teorema se proviamo che $u$ si deve annullare in $P$.

Osserviamo che anche le derivate prime di $u$ sono nulle su $B_{\varepsilon}$. Infatti su $B_{\varepsilon}$, essendo ivi $u=0$, risulta

$$
u_{t t}-u_{y y}=0, \quad \text { ciò } \quad\left(\frac{\partial}{\partial t}-\frac{\partial}{\partial y}\right)\left(\frac{\partial}{\partial t}+\frac{\partial}{\partial y}\right) u=0
$$

e quindi la derivata normale di $u$ è funzione della sola $x$; siccome sullo spigolo $y=t=0$ la normale a un semipiano portante $\mathrm{i}$ dati è tangente all'altro, la derivata normale di $u$ su $B_{\varepsilon}$ è nulla.

Indichiamo con $G$ l'insieme dei punti della frontiera di $T$ non appartenenti ai piani $y= \pm t$. La normale in un qualsiasi punto di $\bar{G}$ forma con l'asse $t$ un angolo maggiore di $\frac{\pi}{4}$. Infatti, posto

$$
\alpha=\frac{\left(x-x_{0}\right)^{2 n-1}}{\varepsilon^{2 n}}, \quad \beta=\frac{(y+t+\varepsilon)^{2 n-1}}{\left(y_{0}+t_{0}+2 \varepsilon\right)^{2 n}}, \quad \gamma=\frac{(y-t+\varepsilon)^{2 n-1}}{\left(y_{0}-t_{0}+2 \varepsilon\right)^{2 n}},
$$

risulta

$$
\begin{gathered}
\beta>\frac{\varepsilon^{2 n-1}}{\left(y_{0}+t_{0}+2 \varepsilon\right)^{2 n}}>0, \quad \gamma \geq \frac{\varepsilon^{2 n-1}}{\left(y_{0}-t_{0}+2 \varepsilon\right)^{2 n}}>0, \text { per } y>|t|, \\
|\cos \widehat{n t}|=\frac{|\beta-\gamma|}{\sqrt{\alpha^{2}+2 \beta^{2}+2 \gamma^{2}}}<\frac{1}{\sqrt{2}} \text { su } \bar{G}
\end{gathered}
$$

Pertanto la superficie $G$ non è caratteristica in alcun punto e vi è un intorno $K$ di $G$ tale che, fissato comunque un polinomio $p$, esiste una solu. 
zione $v$ del problema di CACCHY

$$
L v=p \quad \text { in } \quad K, \quad v=\frac{d v}{d n}=0 \quad \text { su } \quad G\left({ }^{4}\right) .
$$

Notiamo che se $v$ e $w$ sono dne funzioni di classe $C^{(2)}$ in un insieme chiuso $H$, con frontiera $F$ costituita da un numero finito di porzioni di superfici regolari, ed in ogni punto di $F$ o si annulla $v$ con le derivate prime oppure $w$ con le derivate prime si ha

$$
\int_{H}(v L w-w L v) d T=0 .
$$

Supponiamo dapprima $T \subset K$. Fissato un polinomio $p$, sia $v$ la soluzione del problema (3) La frontiera di $T$ è costituita da $G$, ove si annulla $v$ con le sue derivate prime, e da $B_{\varepsilon}$, ove si annulla $u$ con le sue derivate prime. Risulta pertanto

$$
\int_{T} u p d T=0
$$

e per l'arbitrarietà del polinomio $p$ si ha $n \equiv 0$ in $T$.

Nel caso che $T$ non sia contenuto in $K$ si perviene in modo analogo alla dimostrazione del teorema operando una opportuna decomposizione di $T$. Poniamo $K_{0}=K \cap T$ ed indichiamo con $K_{\lambda}$ l'insieme dei punti $(x, y, t)$ con $(x, y+\lambda, t) \in R_{0}$. Esistono $r$ costanti $\lambda_{1}, \lambda_{2}, \ldots, \lambda_{r}$, con $\lambda_{1}>\lambda_{2}>\ldots>\lambda_{r}=0$, tali che $T=\bigcup_{i=1}^{r} K_{\lambda_{i}}$ e l'intersezione di $K_{\lambda_{i}}$ con $T$ non è vuota. Indichiamo con $G_{\lambda}$ l'insieme dei punti $(x, y, t)$ con $(x, y+\lambda, t) \in G$. Fissato comunque un polinomio $p$ esiste una funzione $v$ di classe $C^{(2)}$ in $K_{\lambda_{i}}$, ivi soluzione di $L v=p$, nulla insieme alle sue derivate prime su $G_{\lambda_{i}}$. Pertanto, posto $H_{1}=T \cap K_{\lambda_{1}}$, per le precedenti considerazioni risulta $u=0$ in $H_{1}$. Posto $H_{i}=T \cap\left(K_{\lambda_{i}}-K_{\lambda_{i-1}}\right), i=2,3, \ldots, r$, una volta provato $u=0$ in $H_{i-1}$ segue $u \equiv 0$ in $H_{i}$ perchè $i$ panti della frontiera di $H_{i}$ appartengono o a $B_{\varepsilon}$ o alla frontiera di $H_{i-1}$ (ove $u$ si annulla con le sue derivate prime) oppure a $G_{\lambda_{i}}$ e può essere ripetuto il solito ragionamento.

Questa dimostrazione dell'unicità della soluzione può essere estesa anche a problemi esterni più generali di quello preso in esame. Nello spazio $x, y, t$ consideriamo un arco di curva $\gamma$, opportunamente regolare e l'insieme $E$ dei punti interni a qualche cono con vertice su $\gamma$, asse parallelo all'asse $t$ e

(4) Cfr. ad es. Petrovsky [3], pag. 25. 
ampiezza $\frac{\pi}{4}$. Si potrebbe provare con le stesse precedenti considerazioni che esiste al più una soluzione della equazione delle onde nella regione esterna ad $E$, con valori assegnati sulla frontiera di $E$. L' unicita sussiste anche in piccolo, nell'intorno di ogni punto di $\gamma$. In particolare se un punto $P$ del complementare di $E$ ha proiezioni, $P^{\prime}$ e $P^{\prime \prime}$ sulla frontiera di $\dot{E}$ e $P^{\prime \prime \prime}$ su $\gamma$, univocamente determinate, il valore della soluzione in $P$ dipende dai valori assegnati in an intorno comunque piccolo dei segmenti $P^{\prime} P^{\prime \prime \prime}$ e $P^{\prime \prime} P^{\prime \prime \prime}$ (essi sono segmenti delle due generatrici del cono ohe giacciono sulla frontiera di $E)$.

\section{Dipendenza della soluzione dai dati.}

Esaminiamo ora la dipendenza delle soluzioni della equazione delle onde piane dai dati assegnati sui due piani caratteristici $y= \pm t$. L' esempio seguente serve molto bene a chiarire il problema.

Fissati due numeri naturali $n$ ed $s$, consideriamo la funzione

$$
\begin{aligned}
& u_{n}(x, y, t)=n^{-s \mathfrak{J}_{0}}\left(n \sqrt{t^{3}-y^{2}}\right) \cos n x \text { per } \quad|t| \geq|y| \\
& u_{n}(x, y, t)=n^{-s} I_{0}\left(n \sqrt{y^{2}-t^{2}}\right) \cos n x \text { per } \quad|t| \leq|y|
\end{aligned}
$$

ove $\mathfrak{J}_{0}$ e $I_{0}$ sono le note funzioni di BEsseL. Si verifica facilmente che $u_{n} \grave{\theta}$ soluzione in tutto lo spario del seguente problema:

$$
L u=0, \quad u(x, y, y)=u(x, y,-y)=n^{-s} \cos n x .
$$

Per quanto abbiamo provato relativamente alla regione $|t| \leq|y| e$ per quanto è noto relativamente alla regione $|l| \geq|y|, u_{n}$ è anche l'unica soluzione del problema considerato.

Osserviamo che per $n \rightarrow+\infty$ i dati assegnati sui piani caratteristici convergono uniformemente $a$ zero, $u_{n}$ è pure uniformemente convergente a zero nella regione $|t| \geq|y|$, perchè $\mathfrak{g}_{0} \grave{e}$ limitata su tutto l'asse reale, ma non è convergente a zero in alcun punto della regione esterna $|t|<|y|$.

Infatti per $n \rightarrow+\infty \cos n x$ non converge a zero per aleun valore di $x$ e $n^{-s} I_{0}(n \alpha)$, con $\alpha \neq 0$ diverge a $+\infty$.

Osserviamo che per $n \rightarrow+\infty$ convergono uniformemente a zero non solo i dati ma anche le loro derivate fino all'ordine $s-1$. Per l'arbitrarietà di $s$ ne segne che per il problema «esterno» considerato non vi è dipendenza continua di ordine finito della soluzione dai dati secondo la definizione di HADA. MARD ( $\left.{ }^{5}\right)$; si tratta quindi di un problema non «ben posto » secondo HADAMARD.

(5) Cfr. [2], pagg. 41.46. 


\section{Esistenza della soluzione.}

Osserviamo che una soluzione $u$ della equazione (1), analitica in $x$, prolungata nel piano complesso $x+i \xi$, soddisfa la equazione

$$
u_{t t}+u_{\xi \xi}-u_{y y}=0,
$$

essendo $u_{z_{5}}=-u_{x x}$. Pertanto se $\mathrm{i}$ dati $\varphi_{1}$ e $\varphi_{2}$ sono analitici in $x$ il pro. blema (1), (2) considerato in una regione «esterna $y \geq|t|$ dello spazio $x$, $y, t$ si riconduce con questo prolungamento allo studio del problema in una regione «interna» $y \geq|t|$ dello spazio $\xi, y, t$ consistente nella determina. zione di una soluzione della (4) verificante le condizioni

(5) $\quad u(x+i \xi, y, y)=\varphi_{1}(x+i \xi, y), \quad u(x+i \xi, y,-y)=\varphi_{2}(x+i \xi, y)$.

Per questo problema è ben nota l'espressione della soluzione; essa può essere prolungata analiticamente nello spazio $x, y, t$ fornendo una espressione della soluzione del problema originario.

Precisiamo queste osservazioni in un caso particolare.

Indichiamo con $I$ ed $F$ i seguenti insiemi

$$
\begin{aligned}
& (x, y, t) \in I \quad \text { se } \quad\left|x-x_{0}\right|<\varepsilon, \quad 0<y \pm t<2 a \\
& (x, y) \in F \quad \text { se } \quad\left|x-x_{0}\right| \leq \varepsilon, \quad 0 \leq y \leq a .
\end{aligned}
$$

Siano $\varphi_{1}(x, y), \varphi_{2}(x, y)$ due funzioni di classe $C^{(2)}$ in $F$, fissato $y$ in $(0, a), \varphi_{1} e \varphi_{2}$ siano analitiche rispetto alla variabile $x$ con raggio di convergenza $\varepsilon+2 \sqrt{a^{2}-a y}$. Se inoltre $\varphi_{1}(x, 0)=\varphi_{2}(x, 0)$, esiste una ed una sola funzione $d i$ classe $C^{(2)}$ in $\bar{I}$, analitica rispetto ad $x$, tale che

(6) $\quad L u=0$ in $1 ; \quad u(x, y, y)=\varphi_{1}(x, y), \quad u(x, y,-y)=\varphi_{2}(x, y)$ su $F$.

Dimostriamo il teorema determinando una espressione della soluzione. Indichiamo con $H$ l'insieme dei punti $(x, \xi, y)$ tali che $\mid x-x_{0}<\varepsilon$, $0 \leq y \leq a,|\xi| \leq \varepsilon+2 \sqrt{a^{2}-a y}$. Per le ipotesi fatte le funzioni $\varphi_{1}$ e $\varphi_{2}$ definite in $F$ possono essere prolnngate analiticamente nel piano complesso $x+i \xi$ per ogni $y$ fissato e le funzioni complesse $\varphi_{1}(x+i \xi, y), \varphi_{2}(x+i \xi, y)$, così ottenate, risultano definite in $H$, ivi di classe $C^{(\prime)} \theta$, fissato $y$, olomorfe rispetto alla variabile complessa $x+i \xi$. Pertanto, fissato $x$ in $\left(x_{0}-\varepsilon, x_{0}+\varepsilon\right)$, esiste una ed una sola soluzione di classe $C^{(2)}$ del problema

$$
\begin{gathered}
v_{\xi \xi}+v_{t t}-v_{y y}=0 \quad \text { per } \quad 0<y \pm t<2 a, \quad|\xi|<\varepsilon+\sqrt{(2 a-y)^{2}-t^{2}}, \\
v(\xi, y, y)=\varphi_{1}(x+i \xi, y), \quad v(\xi, y,-y)=\varphi_{2}(x+i \xi, y) \\
\text { per } 0 \leq y \leq a, \quad|\xi| \leq \varepsilon+2 \sqrt{a^{2}-a y .}
\end{gathered}
$$


Posto

$$
w=\log \left\{y-\eta+\left[(y-\eta)^{2}-(\xi-\vartheta)^{2}-(l-\tau)^{2}\right]^{\frac{1}{2}}\right\}-\log \left[(\xi-\vartheta)^{2}+(t-\tau)^{2}\right]^{\frac{1}{2}},
$$

la soluzione $v$ soddisfa alla seguente relazione

$$
\int_{|t|}^{y} \imath(\xi, \lambda, t) d \lambda=\frac{1}{2 \pi} \int_{S}\left(v \frac{d w}{d \nu}-w \frac{d v}{d \nu}\right) d \sigma,
$$

ove $S$ è l'insieme dei punti $(\vartheta, \eta, \tau)$ dei due semipiani $\eta=|\tau|$ interni al cono caratteristico di vertice $(\xi, y, t)$ e $\vee$ è la direzione conormale

$$
\frac{d}{d \nu}=\frac{\sqrt{2}}{2} \frac{\partial}{\partial \eta} \pm \frac{\sqrt{2}}{2} \frac{\partial}{\partial \tau}
$$

col segno + sul semipiano $\eta=\tau$ e col segno - sul semipiano $\eta=-\tau\left({ }^{6}\right)$. Indicato con $S_{r}(r=1,2)$ l'insieme dei punti $(\zeta, \eta)$ tali che

$$
\eta \geq 0, \quad \zeta^{2} \leq(\eta-y)^{2}-\left[t+(-1)^{r} \eta\right]^{2},
$$

il punto $\left(\xi+\zeta, \eta,(-1)^{\prime} \eta\right)$ appartiene ad $S_{r}$ se e soltanto se $(\zeta, \eta)$ appartiene ad $S_{r}$. Posto

$$
w_{r}=\log \left\{y-\eta+\left[(y-\eta)^{2}-\zeta^{2}-\left(t+(-1)^{r} \eta\right)^{2}\right]^{\frac{1}{2}}\right\}-\log \left\{\zeta^{2}+\left[t+(-1)^{r} \eta\right]^{2}\right\}^{\frac{1}{2}},
$$

si ha dalla (7) con facili calcoli

$$
\int_{i t_{1}}^{y} v(\xi, \lambda ; t) d \lambda=\frac{1}{2 \pi} \stackrel{\Sigma}{r=1}_{S_{r}}^{2} \int_{S_{r}} \varphi,[x+i(\xi+\zeta), \eta] \frac{\partial w_{r}}{\partial \eta}-w_{r} \frac{\partial \varphi,[x+i(\xi+\zeta), \eta]}{\partial \eta} d \zeta d \eta
$$

Osserviamo che $v$ dipende anche da $x$ e quindi poniamo $v=v(x, \xi, \lambda, t)$. Notiamo che $S_{\text {, e }} w_{r}$ non dipendono da $x$ e $\xi$; per questo e per le ipotesi

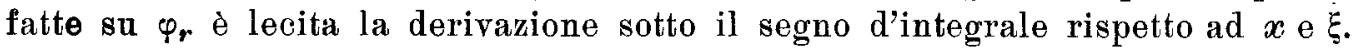
Per la olomorfia delle $\varphi$. risulta

$$
\frac{\partial}{\partial x} \int_{|t|}^{y} v(x, \xi, \lambda, t) d \lambda=\frac{1}{i} \frac{\partial}{\partial \xi} \int_{|t|}^{y} v(x, \xi, \lambda, t) d \lambda
$$

(6) Cfr. ad es. Tricomi [8], pagg. 176-179. Per la esistenza di una soluzione $v$ di classe $C^{(2)}$ vedere HADAMARD [2], pagg. 251.257. 
La funzione $v$, per definizione, è dotata di derivate seconde rispetto a $\xi$ ed $y$ continue e quindi il secondo membro è derivabile rispetto ad $y$ ed è lecito il cambiamento di ordine di derivazione. Ne segue che fissati $y$ e $t$ tali che $0<y \pm t<2 a$, si ha

$\frac{\partial}{\partial x} v(x, \xi, y, t)=\frac{1}{i} \frac{\partial}{\partial \xi} v(x, \xi, y, t) \quad$ per $\quad\left|x-x_{0}\right|<\varepsilon, \quad|\xi|<\varepsilon+2 \sqrt{a^{2}-a y}$, e quindi $v_{\xi \xi}=-v_{x x}$. Posto $u(x, y, t)=v(x, o, y, t)$ la funzione $u$ è la soluzione cercata del problema (6) considerato. Dalle considerazioni svolte segue che essa ha la seguente rappresentazione

(9) $u(x, y, t)=\frac{1}{2 \pi} \sum_{r=1}^{2} \frac{\partial}{\partial y} \int_{S_{r}}\left\{\varphi_{r}(x+i \zeta, \eta) \frac{\partial w_{r}}{\partial \eta}-w_{r} \frac{\partial \varphi,(x+i \zeta, \eta)}{\partial \eta}\right\} d \zeta d \eta$.

Indichiamo una diversa rappresentazione della soluzione ed un'altra possibile dimostrazione della sua esistenza.

Le funzioni $\varphi_{1}$ e $\varphi_{2}$ sono analitiche in $x$ nell'intorno di $x_{0}$ e quindi possiamo porre

$$
\varphi_{1}(x, y)=\sum_{n=0}^{\infty} \alpha_{n}(y)\left(x-x_{0}\right)^{n}, \quad \varphi_{2}(x, y)=\sum_{n=0}^{\infty} \cdot \beta_{n}(y)\left(x-x_{0}\right)^{n},
$$

e risulta $\alpha_{n}(0)=\beta_{n}(0)$ essendo $\varphi_{1}(x, o)=\varphi_{2}(x, o)$.

Sia $u$ la soluzione del problema (6) considerato, analitica in $x$ :

$$
u(x, y, t)=\sum_{n=0}^{\infty} v_{n}(y, t)\left(x-x_{0}\right)^{n}
$$

posto

$$
2 \xi=y+t, \quad 2 \eta=y-t, \quad w_{n}(\xi, \eta)=v_{n}(\xi+\eta, \xi-\eta),
$$

per la (6) e la (11) le $w_{n}$ soddisfano alle seguenti condizioni :

$$
\begin{aligned}
& \frac{\partial^{2} w_{n}}{\partial \xi \partial \eta}=-(n+1)(n+2) w_{n+2}, \\
& w_{n}(\xi, 0)=\alpha_{n}(\xi), \quad w_{n}(0, \eta)=\beta_{n}(\eta),
\end{aligned}
$$

e quindi

$$
w_{n}(\xi, \eta)=\alpha_{n}(\xi)+\beta_{n}(\eta)-\alpha_{n 2}(0)-(n+1)(n+2) \int_{0}^{\xi} d \lambda \int_{0}^{n} w_{n+2}(\lambda, \mu) d \mu .
$$


Con procedimento iterativo si ottiene come presunta espressione delle $w_{n}$

$$
\begin{aligned}
w_{n}(\xi, \eta) & =\sum_{s=0}^{\infty}(-1)^{s} \frac{(n+2 s) !}{n !(s !)^{2}}\left\{s ! \eta^{s} I^{s} \alpha_{n+2 s}(\xi)+\right. \\
& \left.+s ! \xi^{s} I^{s} \beta_{n+2 s}(\eta)-\xi s \eta^{s} \alpha_{n+2 s}(0)\right\}
\end{aligned}
$$

avendo indicato con $I$ l'operatore integrale di RIEMANN-LIOdvILLE:

$$
I^{0} \varphi(z)=\varphi(z), \quad I^{s} \varphi(z)=\int_{0}^{z} \frac{(z-\zeta)^{s-1}}{(s-1) !} \varphi(\zeta) d \zeta, \quad s=1,2, \ldots
$$

Posto

$$
M_{n}=\max _{0 \leq \lambda \leq \rho}\left\{\left|\alpha_{n}(\lambda)\right|+\left|\beta_{n}(\lambda)\right|\right\}
$$

la serie

$$
\sum_{s=0}^{\infty} \frac{(n+2 s) !}{n !(s !)^{2}} 2 M_{n+2 s p^{2 s}}
$$

è maggiorante la serie al secondo membro della (13) per $0 \leq \xi$, $\eta \leq \xi$ e quindi questa serie è convergente se $\rho$ è sufficientemente piccolo per la convergenza delle serie (10). Si potrebbe verificare facilmente che la fun. zione $u$, definita dalla (11) e dalle (10), (12) e (13), è la soluzione del problema (6) considerato.

Anche con questo procedimento si proverebbe l'esistenza della soluzione in un insieme la cui ampiezza dipende dai raggi di convergenza dello sviluppo di TAYLOR rispetto ad $x$ di $\varphi_{1}$ e $\varphi_{2}$. Sembra giustificata la congettura che il campo di esistenza della soluzione dipenda effettivamente da questi raggi di convergenza.

Proviamo infine che nel precedente teorema esistenziale la ipotesi di analiticità rispetto ad $x$ di $\varphi_{1}$ e di $\psi_{2}$ non può essere sostituita dalla ipotesi meno restrittiva che $\varphi_{1}$ e $\varphi_{2}$ siano di classe $C^{(n)}$ rispetto ad $x$, comunque grande sia n. Basta per questo provare che non esiste una soluzione del problema consistente nella ricerca di una funzione $u$ di classe $C^{(2)}$ nell'insieme $Q$ dei punti $(x, y, t)$ tali che $|x| \leq 1,0 \leq y \pm t \leq 1$, verificante in $Q$ le condizioni

$$
u_{t t}-u_{x x}-u_{y y}=0, \quad u(x, y, y)=u(x, y,-y)=|x| x^{2 n-1}
$$


Indicati con $Q_{1}$ e $Q_{2}$ gli insiemi dei punti $(x, y, t)$ con $0 \leq y \pm t \leq 1 \mathrm{e}$ rispettivamente $0 \leq x \leq 1$ e $-1 \leq x \leq 0$, posto

$$
P(x, y, t)=\sum_{s=0}^{n} \frac{x^{2 n-2 s}\left(t^{2}-y^{2}\right)^{s}}{2^{2 s}(2 n-2 s) !(s !)^{2}}
$$

si osserva che la (14) è soddisfatta da $P$ in $Q_{1}$ e da $-P$ in $Q_{2}$. Se esistesse in $Q$ una soluzione $u$ della (14) per il teorema di unicità provato si avrebbe $u=P$ in $Q_{1}$ ed $u=-P$ in $Q_{2}$; quindi per la continuità di $u$ dovrebbe essere $P(o, y, t) \equiv-P(o, y, t)$ che è assurdo non essendo $P(o, y, t)$ identicamente uguale a zero.

Osserviamo che queste considerazioni non possono essere ripetute per il problema interno relativo alla regione $t \pm y>0$ perchè in questo caso l' unicità è assicurata in una regione delimitata da superfici caratteristiche.

\section{BIBLIOGRAFIA}

[1] D'ADHÉmaR, R., Les équations aux dérivées partielles à caractéristiques réelles, . Colle. ction Scientia", 29, 1907.

[2] HadamaRd, J, Le problème de Cauchy, Paris, Hermann, 1932.

[3] Petrovsix, X. G., Lectures on partial differential equations, Interscience Publișhers, New York, 1954.

[4] Ruesz, M., L'intégrale de Riemann-Liouville et le problème de Cauchy, "Acta Math.», 81, 1.223, (1949).

[5] - - Problems related to characteristic surfaces, "Proceedings of the conference on Differential Equations », University of Maryland (1956), 57.72.

[6] Temoxarov, A. A. Soluzioni analitiche della equazione delle onde piane, \& Moskov. Oblast. Pedagog. Inst. Uc. Zap. Trudy Kafedr Mat.», 20 (1954). 17.36 (in russo).

[7] Temuxakov, A. A. e SoHoAn A. M., Soluzioni analitiche di un certo problema di Goursat, - Moskov. Oblast. Pedagog Inst. Ue. Zap. Trudy Kafedr Mat », 21 (1950), 23 -33 (in russo).

[8] Tricom F, Equazioni a derivate parziali, Cremonese, Roma (1957).

[9] Volterra, V., Sur les vibrations des corps élastiques isotropes, "Acta Math. ", 18 (1894), 161-232. 